Article

\title{
Cracked, Porous Rocks and Fluids: Moon and Earth Paradox
}

\author{
Jan V.M. Borgomano * (D), Jérôme Fortin and Yves Guéguen \\ Laboratoire de Géologie, Ecole Normale Supérieure/CNRS, UMR8538, PSL Research University, \\ 75005 Paris, France; fortin@geologie.ens.fr (J.F.); gueguen@geologie.ens.fr (Y.G.) \\ * Correspondence: borgomano@geologie.ens.fr
}

Received: 14 October 2019; Accepted: 7 November 2019; Published: 9 November 2019

\begin{abstract}
Elastic wave velocities are key parameters in geosciences. In seismology at a large scale, or in seismic exploration at a more local and shallower scale, they were the main source of information for a long time. At the time of the Apollo mission, Anderson explained the unexpected result of very low velocities in Moon surface rocks by an intense cracking resulting from meteoritic impacts. Yet, it was also known that the $Q$ factor was high. This could appear as a paradox. In the shallow layers of the Earth, rocks are porous. These shallow layers are of major importance in the Earth since they contain fluids. This is why velocities are higher and Q values lower in the Earth's shallow layers than in the Moon's shallow layers. Cracks have a determining effect on elastic properties because they are very compliant. Fluids also play a key role. Combining poroelasticity and effective elasticity, two independent theories much developed since the time of the Apollo mission, makes it possible to revisit the contrasting results observed in the Moon case and in the Earth case. Experimental results obtained on cracked synthetic glass show that dry cracks result in a strong decrease in velocity. On the other hand, saturated porous limestones exhibit a strong frequency-dependent attenuation when thermally cracked. The presence of fluid is the key factor.
\end{abstract}

Keywords: rock physics; pores; cracks; fluids; effective medium theory; poroelasticity

\section{Introduction}

In general, the heterogeneity of crustal rocks is mainly the result of variable mineral composition and of the presence of pores and cracks. At all scales, from local to regional, rocks are heterogeneous. The influence of heterogeneity in rock, as long as it remains moderate, can often be handled by considering that the medium is statistically homogeneous on the local scale. That means that a representative volume element (RVE) exists and that any part of the system with a volume much larger than the RVE has identical physical properties.

Within this assumption, use of effective elasticity in order to predict elastic properties and, hence, elastic wave velocities is of direct interest. In shallow conditions, rocks contain pores and cracks. These "defects" were identified for a long time as having a major influence on elastic properties.

When fluids are present, frequency dependence is expected, and the combined use of effective elasticity and poroelasticity allows accounting for it. This can be applied to the results described a long time ago by Anderson [1], who reported that the average sound velocity of Moon rocks was close to provolone cheese and very low in comparison to those found on Earth (Figure 1). 


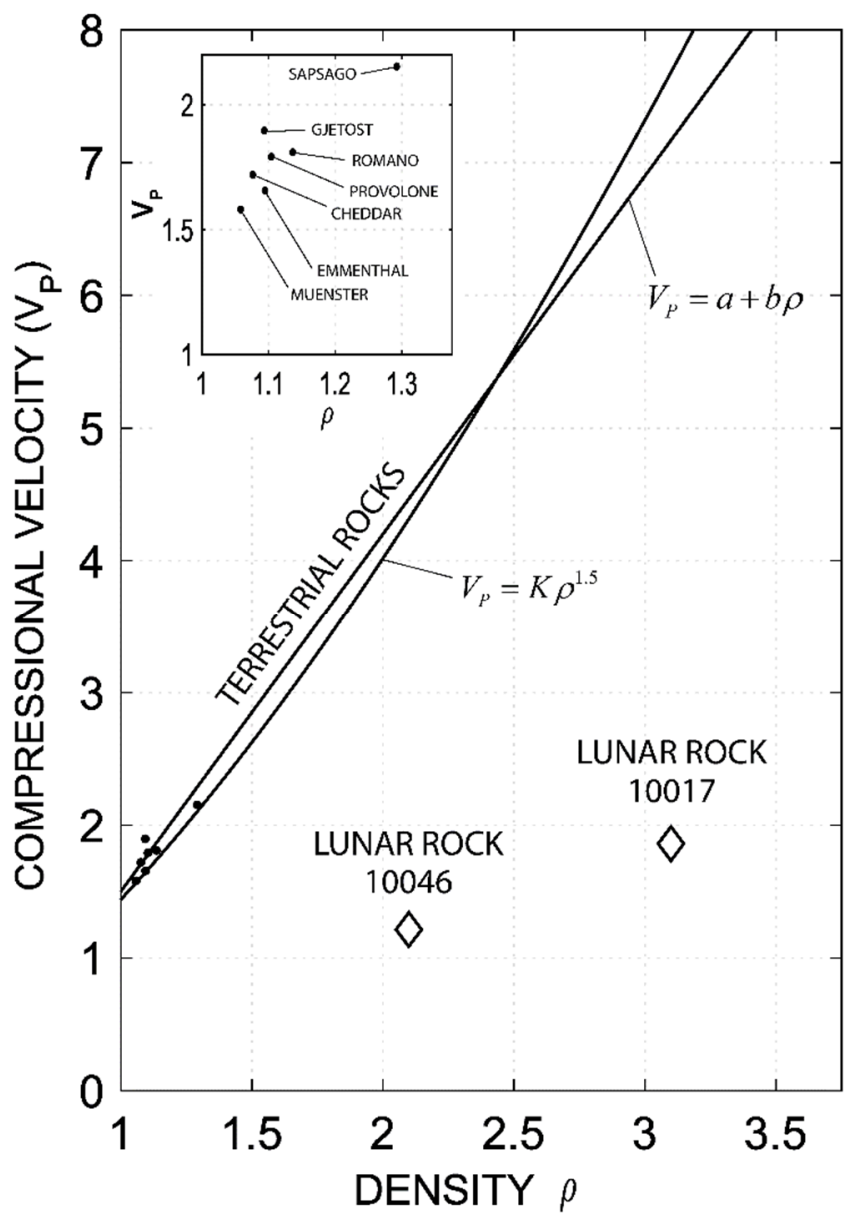

Figure 1. Comparison between sound velocities for lunar rocks and for various earth materials, from Schreiber and Anderson [1].

\section{Effective Elasticity of Cracked Rocks}

Effective elasticity allows one to derive the elastic moduli for a dry or saturated cracked rock in terms of the parameters defined below. Let us consider the simple, but interesting case of an isotropic distribution of three-dimensional (3D) identical circular cracks of radius $r$ and aspect ratio $\zeta$ (thickness/diameter). The non-cracked matrix is assumed to be isotropic. Three groups of parameters are needed: (1) $E_{0}$, Young modulus (or equivalently $K_{0}$, the bulk modulus, or $G_{0}$, the shear modulus) and $v_{0}$ Poisson ratio of the isotropic matrix; (2) $K_{f}$, the fluid bulk modulus (the fluid is taken below as liquid water); (3) crack density $\rho=n r^{3} / V$, if an REV of volume $V$ contains $\mathrm{n}$ cracks of radius $r$ (this non-dimensional parameter is expected to vary between 0 and 1 , but the model under the assumption of non-interactive cracks is accurate only if $\rho$ is low enough). Because cracks are very compliant, the effect of dry cracks on the modulus is very strong. The bulk modulus is decreased in the dry case (compared to the intact matrix modulus), and so is the shear modulus. Within the approximation of very thin cracks $\left(\zeta<10^{-3}\right)[2,3]$, the following relationships exist:

$$
\frac{K_{0}}{K_{d r y}}=1+\frac{16\left(1-v_{0}^{2}\right)}{9\left(1-2 v_{0}\right)} \rho \text { and } \frac{G_{0}}{G_{d r y}}=1+\frac{32\left(1-v_{0}\right)\left(5-v_{0}\right)}{45\left(2-v_{0}\right)} \rho,
$$

where $K_{d r y}$ stands for the dry cracked rock bulk modulus (same notation for $G$ ). Using a standard value $v_{0}=0.25$, the above equations become $K_{0} / K_{d r y}=1+3.3 \rho$ and $G_{0} / G_{d r y}=1+1.45 \rho$.

For a water-saturated rock, the effect is less important for the bulk modulus, and more important for the shear modulus. Note that, in (high-frequency) effective elasticity, fluid phases are considered to 
be immobile. This means that different cracks may experience different fluid pressures since fluid has no time to move. This justifies the name "unrelaxed" for the effective saturated moduli. Experimentally, the appropriate measurements are ultrasonic measurements, because they are very-high-frequency ones, so that the "unrelaxed" situation is obtained. Then, with the same approximations as above [2,3], the following relationships exist:

$$
\frac{K_{0}}{K_{u r}}=1 \text { and } \frac{G_{0}}{G_{u r}}=1+\frac{32\left(1-v_{0}\right)}{15\left(2-v_{0}\right)} \rho,
$$

where $K_{u r}$ stands for the unrelaxed saturated cracked rock bulk modulus (same notation for $G$ ). Using a standard value $v_{0}=0.25$, the above equation becomes $G_{0} / G_{u r}=1+0.91 \rho$.

Saturation strongly modifies the dry results; the unrelaxed saturated bulk modulus has, under the assumption of thin cracks, the same value as that of the intact matrix, but the saturated shear modulus is decreased and different from the dry case. Using values reported by Anderson [1], (dry rock P-wave velocity of the order of $2 \mathrm{~km} / \mathrm{s}$ for lunar rocks instead of $6 \mathrm{~km} / \mathrm{s}$ for Earth rocks), it is straightforward to check that $\rho$ should be higher than 1 . The crack density is so high that the above model under the approximation of non-interactive cracks is no longer quantitative, but the implication is that the cracking is very intense. A very useful (but unavailable) complementary set of data would be the velocity variation of lunar rocks with pressure. Since cracks close under pressure, one expects a strong decrease in crack density at higher pressure, down to a crack density range where the above model would be quantitatively applicable [2,3]. Differential [4,5] or self-consistent schemes [6] may also be used beyond the limits of applicability of the non-interaction approximation.

As Anderson pointed out, this shows that lunar rocks are highly cracked and dry. If not dry, they would exhibit a much higher bulk modulus and, hence, a much higher P-wave velocity.

\section{Effective Elasticity of Porous Rocks}

The situation is very different for terrestrial rocks. They can contain cracks, but with a much lower crack density. Most importantly, in the crust, sedimentary rocks are porous and not dry. Pores are not very compliant because they are approximately round-shaped. Then, the bulk modulus and the shear modulus can be expressed approximately, in the dry case usually, in terms of porosity $\Phi[2,3]$ as follows:

$$
\frac{K_{0}}{K_{d r y}}=1+\frac{3\left(1-v_{0}\right)}{2\left(1-2 v_{0}\right)} \Phi \text { and } \frac{G_{0}}{G_{d r y}}=1+\frac{15\left(1-v_{0}\right)}{7-5 v_{0}} \Phi .
$$

Using a standard value $v_{0}=0.25$, the above equations become $K_{0} / K_{d r y}=1+2.25 \Phi$ and $G_{0} / G_{d r y}=$ $1+1.9 \Phi$.

For the saturated case, unrelaxed moduli are determined as follows:

$$
\frac{K_{0}}{K_{u r}}=1 \text { and } \frac{G_{0}}{G_{u r}}=1+\frac{15\left(1-v_{0}\right)}{7-5 v_{0}} \Phi .
$$

In contrast with the previous case (cracks), saturation does not modify the shear modulus compared to the dry case. However, the most important result is that the effect of pores is less important than that of cracks.

\section{Poroelasticity}

As before, we consider a cracked isotropic rock, with an isotropic distribution of identical cracks. This is a particular case of a porous medium, with a low porosity. The cracks are assumed to be connected and water-saturated. Pores can be present as well. Then, porosity is higher, and cracks and pores are assumed to be connected. For simplification, we consider below a case where only cracks are present. 


\subsection{Relaxed (Isobaric) Moduli}

The assumption is that the fluid is isobaric within an RVE (a reasonable assumption at seismic frequencies that are low enough). This is a different case from the one examined just above. Poroelasticity applies [3]. The situation we are interested in is such that there is no macroscopic fluid flow between RVEs, but the fluid pressure varies from one RVE to another. The bulk and shear moduli are called in that case the undrained bulk and shear moduli $K_{u}$ and $G_{u}$. They can be measured under any condition where there is not enough time for the macroscopic flow to occur from one RVE to another. Then, the following relationships apply:

$$
\frac{K_{0}}{K_{u}}=1 \text { and } \frac{G_{0}}{G_{d r y}}=1+\frac{32\left(1-v_{0}\right)\left(5-v_{0}\right)}{45\left(2-v_{0}\right)} \rho \text { and } \frac{G_{d r y}}{G_{u}}=1 .
$$

This simple result means that cracks are "invisible" in that case for the bulk modulus. The cracks affect only the shear modulus.

\subsection{Unrelaxed Moduli}

If wavespeed measurements are performed at ultrasonic frequencies, typical of laboratory experiments, the RVE is not isobaric. This case is out of the validity range of poroelasticity since fluid pressure is variable within an RVE. Of course, it is well within the validity range of effective elasticity as described above (Equation (2)). Above a certain critical frequency, any experimental measurement of wavespeeds corresponds to this non-isobaric situation. There exists a transition domain between regimes of "low" (relaxed) and "high" (unrelaxed) frequency.

A key question regards the critical frequency $f_{c}$ value. In fluid-saturated cracked rocks, the effect is called "squirt flow". The critical frequency of "squirt flow" (or local fluid flow) $f_{c}$ is obtained by calculating the time needed for local fluid motion between two neighbor cracks, as due to local pressure gradients [7].

$$
f_{c} \sim \frac{\zeta^{3} E_{0}}{20 \eta}
$$

where, typically, the crack aspect ratio $\zeta$ is $\sim 10^{-3}$, Young's modulus $E_{0}$ is $\sim 70 \mathrm{GPa}$, and water viscosity $\eta$ is $\sim 10^{-3} \mathrm{~Pa} \cdot \mathrm{s}$, resulting in $f_{c} \sim 3.5 \mathrm{kHz}$. This means that, typically, in cracked saturated rocks, one expects to get the "unrelaxed" moduli from ultrasonic data, and the "relaxed" ones from seismic data.

\section{5. $Q$ Factor}

It results from the above that, in a saturated cracked medium, wavespeeds are expected to be frequency-dependent. If we use a viscoelastic framework [8], the frequency dependence effect is accompanied by dispersion and attenuation, following the Kramers-Kronig relationships [9].

In the simple case considered above, the dissipation is in shear only. Using the previous parameter values, one gets the following:

$$
\frac{\Delta G}{G}=0.54 \rho,
$$

where $\Delta G$ stands for the maximum variation of $G$ (from the unrelaxed state to the relaxed one, in a water-saturated cracked rock).

In the approximation of the simple linear viscoelastic body, $Q^{-1}{ }_{\max }=0.5 \Delta G / G$, so that the squirt flow effect is expected to produce an attenuation peak on shear waves at $f_{c}$ of

$$
Q_{\max }^{-1}=0.27 \rho,
$$

and, similarly, will also produce some attenuation for the P-wave $\left(Q^{-1} \max =0.09 \rho\right)$. 
For a crack density of 0.2 (likely to be lower than that for Moon rocks, but in a range where the above models are approximately valid), one gets an attenuation peak of $Q^{-1}$ max $=0.05$ for the shear wave $\left(Q^{-1}\right.$ max $=0.02$ for the P-wave). This is a high value, possible only if fluids are present.

The above model can be extended to porous rocks. In that case, it can be shown that crack-pore flow also induces a strong bulk effect [10].

In the situation of dry cracks, no dissipation related to fluid flow is expected and Equation (1) would apply both at low and high frequencies. In that case, $\Delta G / G=0$ and $\Delta K / K=0$, which results in $Q^{-1}$ max $=0$, regardless of the crack density. This explains the apparent paradox between Moon (high crack density, low attenuation) and Earth rocks (low crack density, high attenuation).

\section{Experimental Data}

Convenient examples to illustrate the above predictions are given by data obtained on glass and Indiana limestone. A homogenous glass can be quenched, resulting in a connected network of cracks (Figure 2a) [11]. As with most sedimentary rocks, Indiana limestone is porous (Figure 2b). Its crack content can be modified by thermal cracking, but its porosity is a characteristic of the rock that cannot be modified easily. The fact that pores are present is important. It implies that squirt flow can take place not only from crack to crack like in the glass, but as also from crack to pore, resulting in bulk dispersion together with shear dispersion [10]. Here, we present a glass sample that was quenched from a temperature of $300{ }^{\circ} \mathrm{C}$, and an Indiana limestone that was heated up to $500{ }^{\circ} \mathrm{C}$ for an hour prior to a natural cooling.

(a)

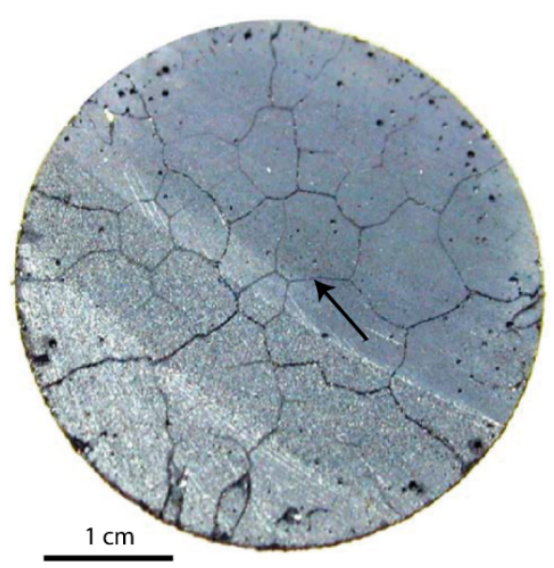

(b) Cracked Indiana limestone

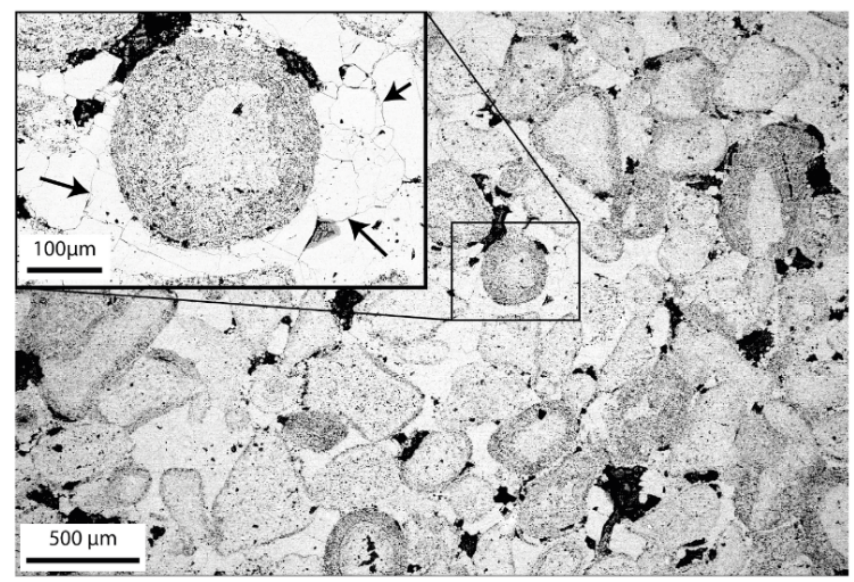

Figure 2. (a) Surface photography of cracked glass [12]. (b) SEM photomicrograph of Indiana limestone [13]. Arrows show examples of thermally induced cracks.

As seen on the microstructures (Figure 2), the glass was composed of a network of cracks that could be of a millimetric to centimetric scale (Figure 2a), whereas, in the Indiana limestone, the cracks were essentially induced in the homogeneous intergranular cement, with sizes around $100 \mu \mathrm{m}$ (Figure 2b). The crack porosity of the thermally treated (TT) glass was measured around $0.24 \%$ using a mercury porosimeter [14]. Overall, the Indiana sample bears a total porosity of $\Phi=11.4 \%$, measured by the triple-weight method [13], essentially distributed between intragranular micropores and some intergranular mesopores (Figure 2b).

Permeability of the cracked glass was measured using the pulse decay method with argon and water [14], and it was found to decrease strongly from $8 \times 10^{-17}$ to $4 \times 10^{-21} \mathrm{~m}^{2}$ with an increase in effective pressure from 2 to $20 \mathrm{MPa}$ (Figure 3a). This is characteristic of crack closure with pressure, since the hydraulic conductivity is solely controlled by crack porosity. On the other hand, the permeabilities of the intact and the cracked Indiana limestones, measured with the steady-state flow rate method with water, exhibited a much smaller variation with effective pressure, decreasing from an average 
of $2.3 \times 10^{-17}$ to $1.4 \times 10^{-17} \mathrm{~m}^{2}$ (Figure $3 \mathrm{~b}$ ). We can note that the results for the intact and cracked limestone differed negligibly, leading to the belief that the pores were the main contributor to the hydraulic conductivity, with a very small contribution of the microcracks (Figure 3b).

(a) Cracked glass

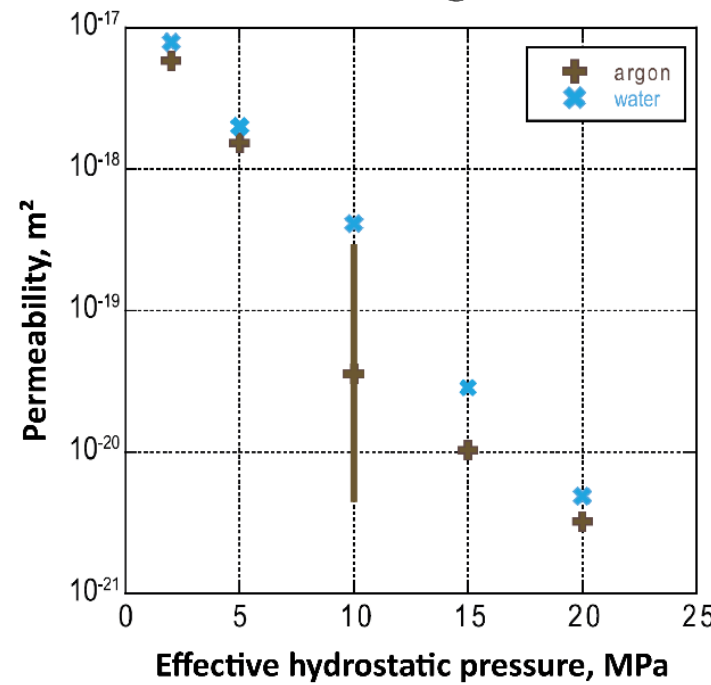

(b) Indiana limestone

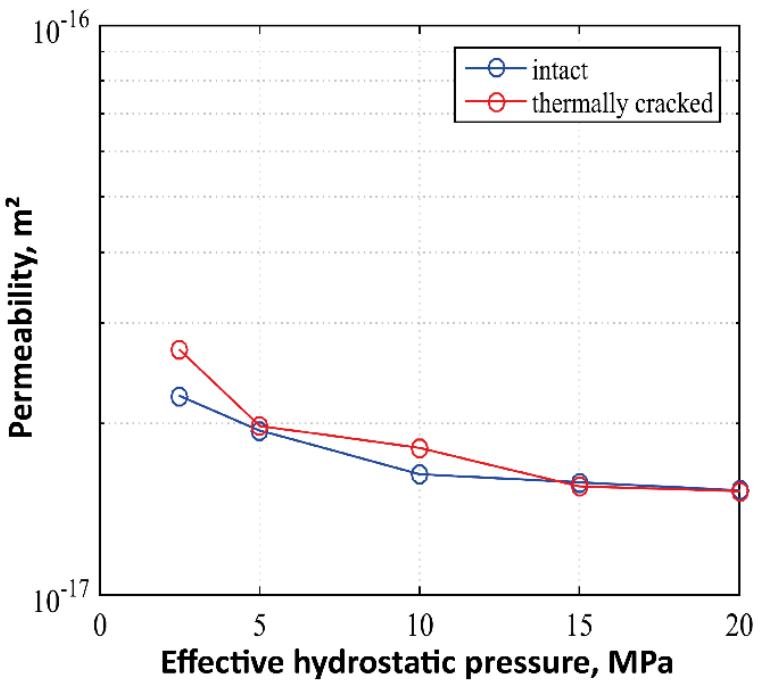

Figure 3. (a) Permeability as a function of effective pressure for the cracked glass [14] and (b) for the intact and cracked Indiana limestone [13].

Results of the elastic/acoustic properties are presented in Figure 4. For the glass sample, ultrasonic $\mathrm{P}$ - and S-wave velocities were measured over a range of effective pressures from 2.5 to $20 \mathrm{MPa}$ [14] (Figure 4a). The intact sample exhibited no pressure dependence. For the cracked sample in dry conditions, the P- and S-wave velocities increased with pressure, consistently with crack closure. However, the water-saturated cracked sample exhibited no pressure dependence, and velocities were close to the intact sample (Figure 4a). This is consistent with the high-frequency unrelaxed regime when crack-to-crack squirt flow occurs (Equation (2)).

(a)
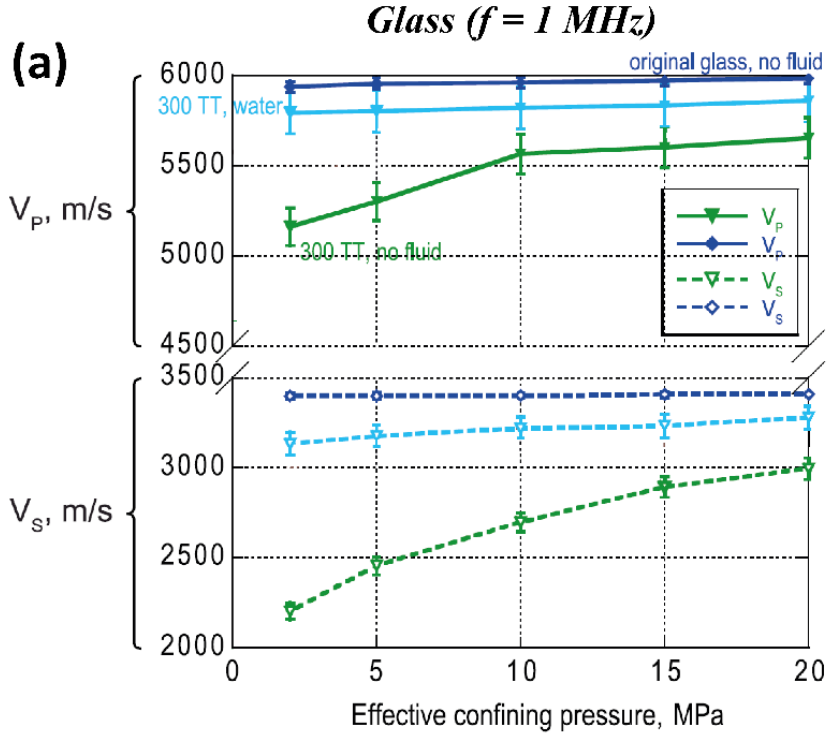

(b)
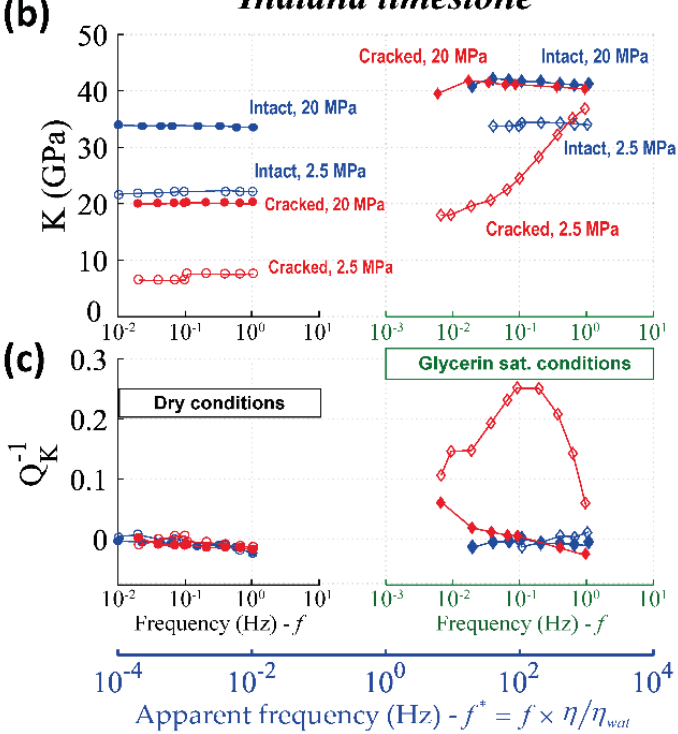

Figure 4. (a) Ultrasonic P- and S-wave velocities on the glass sample, intact and thermally cracked (TT) [14]. (b) Bulk dispersion and (c) attenuation of the intact and cracked Indiana limestone, at effective pressures of 2.5 and $20 \mathrm{MPa}$. The samples were tested in dry and fluid-saturated conditions. "Apparent frequency" is frequency normalized by the fluid's viscosity, using water as a reference [15]. 
The Indiana limestone was investigated using a specific apparatus at École normale supérieure (ENS) Paris that measures the frequency dispersion/attenuation of elastic moduli over a broad frequency range $(0.004-100 \mathrm{~Hz})$ using the forced-oscillation method [13,15]. Here, the results of the bulk modulus dispersion and attenuation $\left(Q_{K}^{-1}\right)$ are presented (Figures $4 \mathrm{~b}$ and $4 \mathrm{c}$, respectively), as induced from pure hydrostatic conditions over an experimental frequency range of $0.004-1 \mathrm{~Hz}$ and effective pressures of 2.5 and $20 \mathrm{MPa}$. The frequency was normalized by the dynamic viscosity of the saturating fluid/gas $(\eta)$, using water as a reference, in order to visualize the results in terms of "apparent frequency" $\left(f^{*}=\right.$ $\left.f \times \eta / \eta_{w a t}\right)$. With the use of glycerin $\left.\eta_{g l y}=1000 \mathrm{mPa} \cdot \mathrm{s}\right)$ instead of water $\left(\eta_{w a t}=1 \mathrm{mPa} \cdot \mathrm{s}\right)$, one can investigate the bulk dispersion/attenuation in the apparent seismic frequency range (4-1000 Hz) of a water-saturated rock (Figure $4 b, c)$, as long as solely viscous-driven fluid-flow processes occur such as squirt flow (Equation (6)).

Both the intact and cracked Indiana samples were non-dispersive in the absence of fluid and showed no attenuation (Figure 4b,c); the glycerin-saturated intact sample also showed no attenuation. On the other hand, the glycerin-saturated cracked sample at low effective pressure (2.5 MPa) exhibited a strong bulk dispersion (Figure $4 \mathrm{~b}$ ) in the seismic range, associated with a strong attenuation peak (Figure 4c) around $100 \mathrm{~Hz}$. This dispersion/attenuation was absent for a high effective pressure (20 MPa), related to crack closure. Therefore, the observed dispersion was likely to be related to crack-to-pore squirt flow, with a transition between the relaxed $(<10 \mathrm{~Hz})$ and unrelaxed regimes $(>1000 \mathrm{~Hz})$. Similarly to the glass sample, the "unrelaxed" elastic properties of the cracked limestone had low sensibility to the effective pressure, contrarily to the dry case.

One may ask if the cut-off frequency of the squirt flow may be predicted. In order to use the

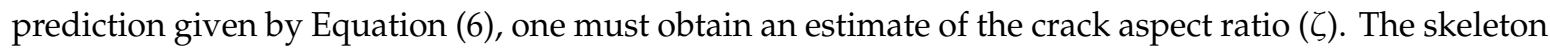
parameters of the glass were given by the intact sample $\left(E_{0}=84 \mathrm{GPa}, v_{0}=0.27\right)$, and, for the Indiana limestone, one can take the properties of calcite in carbonates $\left(E_{0}=83 \mathrm{GPa}, v_{0}=0.32,[16]\right)$. The pressure closure of a crack is given by $P_{\text {close }}=E_{0} \pi \zeta /\left(4\left(1-v_{0}^{2}\right)\right)$ [17], and the valu was found to be around $20 \mathrm{MPa}$ for both the glass and the Indiana limestone, which would give an aspect ratio around $\zeta \approx 2.8 \times 10^{-4}$ for both samples. Therefore, the predicted cut-off frequency for squirt flow (Equation (6)) would be around $92 \mathrm{~Hz}$, under water-saturated conditions $\left(\eta=10^{-3} \mathrm{~Pa} \cdot \mathrm{s}\right)$, which seems in agreement with the results obtained on the cracked limestone (Figure $4 \mathrm{~b}, \mathrm{c})$.

One interesting alternative method to determine $\zeta$ is to use the pressure dependence of the permeability. In a cracked medium, permeability may be calculated as follows [18]:

$$
k=k_{0} e^{-a P},
$$

where $k_{0}$ is the unconfined permeability. Moreover, the aperture $(w)$ of the crack varies with pressure according to Equation (10) [19].

$$
w \sim w_{0}\left(1-\frac{P}{E_{0} \zeta}\right)
$$

where $w_{0}$ is the unconfined aperture. If we assume that the variations of $k$ and $w^{3}$ with pressure are proportional, from Equations (9) and (10), we obtain $a=3 /\left(E_{0} \zeta\right)$. This method applies only to a cracked medium and not to a mixture of cracks and pores. For the glass sample, the slope of the permeability versus pressure in Figure 3 a (considering the log scale) gives us $a \approx 1.8 \times 10^{-7}$, from which we finally obtain $\zeta=3 /\left(E_{0} a\right) \approx 2 \times 10^{-4}$, consistent with the previous method.

\section{Conclusions}

Simple models of isotropic cracked dry rocks show that the elastic wave velocities can decrease substantially if crack density is high. However, P-wave velocities are not really affected in the saturated case (because only the shear modulus is modified). Shear attenuation is predicted if cracked rocks are saturated. This fits well with Moon surface rocks, where a strong velocity decrease is expected for dry cracked rocks, but no strong attenuation. 
In the Earth case, rocks are often saturated but crack density is low, implying a small velocity decrease. Attenuation and frequency-dependent behavior are expected because of the fluid and the possible presence of pores. Experimental results documented the squirt-flow effect.

Effective elasticity and poroelasticity provide a satisfactory theoretical background to explain these contrasting situations, and revisit the results obtained long ago by Anderson.

Author Contributions: Conceptualization, J.V.M.B., J.F. and Y.G.; validation, J.V.M.B.; formal analysis, J.V.M.B.; investigation, J.V.M.B.; data curation, J.V.M.B.; writing-original draft preparation, J.V.M.B. and Y.G.; writing-review and editing, J.F.; visualization, J.V.M.B.; supervision, J.F. and Y.G.; project administration, J.F.; funding acquisition, J.F.

Funding: This project was supported by TOTAL, under project number FR00007429.

Conflicts of Interest: The author declares no conflicts of interest.

\section{References}

1. Schreiber, E.; Anderson, O.L. Properties and composition of lunar materials: Earth analogies. Science 1970, 168, 1579-1580. [CrossRef] [PubMed]

2. Fortin, J.; Guéguen, Y.; Schubnel, A. Effect of pore collapse and grain crushing on ultrasonic velocities and Vp/Vs. J. Geophys. Res. 2007, 112, B08207. [CrossRef]

3. Guéguen, Y.; Kachanov, M. Effective elastic properties of cracked and porous rocks. In Mechanics of Crustal Rocks, CISM Courses and Lectures; Leroy, Y., Lehner, F., Eds.; Springer: Vienna, NY, USA, 2011; Volume 533, pp. 73-126.

4. Berryman, J.G.; Pride, S.R.; Wang, H.F. A differential scheme for elastic properties of rocks with dry or saturated cracks. Geophys. J. Int. 2002, 151, 597-611. [CrossRef]

5. Hashin, Z. The differential scheme and its application to cracked materials. J. Mech. Phys. Solids 1988, 36, 719-734. [CrossRef]

6. Budiansky, B.; O'Connell, R.J. Elastic moduli of a cracked solid. Int. J. Solids Struct. 1976, 12, 81-97. [CrossRef]

7. O'Connell, R.J.; Budiansky, B. Viscoelastic properties of fluid-saturated cracked solids. J. Geophys. Res. 1977, 82, 5719-5735. [CrossRef]

8. Pimienta, L.; Fortin, J.; Guéguen, Y. Effect of fluids and frequencies on Poisson's ratio of sandstone samples. Geophysics 2016, 81, D183-D195. [CrossRef]

9. O'Connell, R.J.; Budiansky, B. Measurements of dissipation in viscoelastic media. Geophys. Res. Lett. 1978, 5, 5-8. [CrossRef]

10. Adelinet, M.; Fortin, J.; Guéguen, Y. Dispersion of elastic moduli in a porous-cracked rock. Tectonophysics 2011, 503, 173-181. [CrossRef]

11. Mallet, C.; Fortin, J.; Guéguen, Y.; Bouyer, F. Effective elastic properties of cracked solids: An experimental investigation. Int. J. Fract. 2013, 182, 275-282. [CrossRef]

12. Mallet, C.; Fortin, J.; Guéguen, Y.; Bouyer, F. Brittle creep and subcritical crack propagation in glass submitted to triaxial conditions. J. Geophys. Res. Solid Earth 2015, 120, 879-893. [CrossRef]

13. Borgomano, J. Dispersion of the Elastic Moduli of Saturated Carbonate Rocks: Experimental Study and Modelisation. Ph.D. Thesis, PSL Research University, Paris, France, 2018. Available online: https:/tel. archives-ouvertes.fr/tel-01892045v2 (accessed on 30 June 2019).

14. Ougier-Simonin, A.; Guéguen, Y.; Fortin, J.; Schubnel, A.; Bouyer, F. Permeability and elastic properties of cracked glass under pressure. J. Geophys. Res. Solid Earth 2011, 116, 1-12. [CrossRef]

15. Borgomano, J.V.M.; Pimienta, L.; Fortin, J.; Guéguen, Y. Dispersion and attenuation measurements of the elastic moduli of a dual-porosity limestone. J. Geophys. Res. Solid Earth 2017, 122, 2690-2711. [CrossRef]

16. Mavko, G.; Mukerji, T.; Dvorkin, J. The Rock Physics Handbook: Tools for Seismic Analysis of Porous Media; Cambridge University Press: Stanford, CA, USA, 2009.

17. Walsh, J.B. The effect of cracks on the compressibility of rock. J. Geophys. Res. 1965, 70, 381-389. [CrossRef] 
18. Brace, W.F.; Walsh, J.B.; Frangos, W.T. Permeability of granite under high pressure. J. Geophys. Res. 1968, 73, 2225-2236. [CrossRef]

19. Jaeger, J.C.; Cook, N.G.; Zimmerman, R. Fundamentals of Rock Mechanics; Blackwell Pub.: Malden, MA, USA, 2007.

(C) 2019 by the authors. Licensee MDPI, Basel, Switzerland. This article is an open access article distributed under the terms and conditions of the Creative Commons Attribution (CC BY) license (http://creativecommons.org/licenses/by/4.0/). 